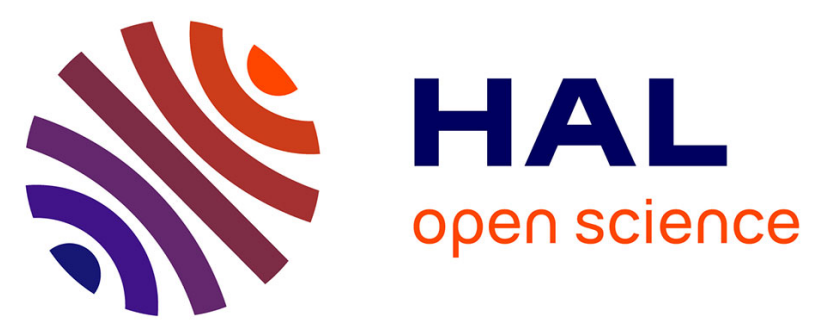

\title{
Multivariate texture retrieval using the geodesic distance between elliptically distributed random variables
}

\author{
Lionel Bombrun, Yannick Berthoumieu, Nour-Eddine Lasmar, Geert
}

Verdoolaege

\section{- To cite this version:}

Lionel Bombrun, Yannick Berthoumieu, Nour-Eddine Lasmar, Geert Verdoolaege. Multivariate texture retrieval using the geodesic distance between elliptically distributed random variables. 18th IEEE International Conference on Image Processing (ICIP), 2011, Bruxelles, Belgium. pp.3637 3640, 10.1109/ICIP.2011.6116506 . hal-00661686

\section{HAL Id: hal-00661686 \\ https://hal.science/hal-00661686}

Submitted on 23 Jan 2012

HAL is a multi-disciplinary open access archive for the deposit and dissemination of scientific research documents, whether they are published or not. The documents may come from teaching and research institutions in France or abroad, or from public or private research centers.
L'archive ouverte pluridisciplinaire HAL, est destinée au dépôt et à la diffusion de documents scientifiques de niveau recherche, publiés ou non, émanant des établissements d'enseignement et de recherche français ou étrangers, des laboratoires publics ou privés. 


\title{
MULTIVARIATE TEXTURE RETRIEVAL USING THE GEODESIC DISTANCE BETWEEN ELLIPTICALLY DISTRIBUTED RANDOM VARIABLES
}

\author{
Lionel Bombrun ${ }^{1}$, Yannick Berthoumieu ${ }^{1}$, Nour-Eddine Lasmar ${ }^{1}$ and Geert Verdoolaege ${ }^{2}$ \\ ${ }^{1}$ : Université de Bordeaux, Laboratoire IMS, UMR 5218 \\ Groupe Signal et Image, 351 cours de la libération, 33402 Talence \\ \{lionel.bombrun, yannick.berthoumieu,nour-eddine.lasmar\}@ims-bordeaux.fr \\ 2 : Department of Applied Physics - Ghent University - Belgium, geert.verdoolaege@ugent.be
}

\begin{abstract}
This paper presents a new texture retrieval algorithm based on elliptical distributions for the modeling of wavelet subbands. For measuring similarity between two texture images, the geodesic distance (GD) is considered. A closed form for fixed shape parameters and an approximation when assuming the geodesic coordinate functions as straight lines are given. Taken into various elliptical choices, the multivariate Laplace and $\mathcal{G}^{0}$ distributions are introduced for modeling respectively the color cue and spatial dependencies of the wavelet coefficients. A multi-model classification approach is then proposed to combine the similarity measures.

A comparative study between some multivariate models on the VisTex image database is conducted and reveals that the combination of the multivariate Laplace modeling for the color dependency and the multivariate $\mathcal{G}^{0}$ modeling for spatial one achieves higher recognition rates than other approaches.
\end{abstract}

Index Terms - Texture, Geodesic distance, Multivariate elliptical distribution.

\section{INTRODUCTION}

In the context of texture image recognition, the wavelet representation domain has been widely considered to characterize the texture. The multiscale recognition algorithm is composed by two main steps. One is the feature extraction which consists in modeling each wavelet subband by a given probability density function (pdf). Its estimated parameters compose the signature of the texture. Then, a similarity measure based on a probabilistic metric is computed between the query image and all the other images of the database.

Many univariate models such as the Generalized Gaussian [1] and the Weibull [2] distributions have been proposed to characterize the wavelet subbands. Those approaches are quite simple but they do not fully exploit the texture information. Multivariate distributions have recently been introduced to model the spatial and/or color dependencies of the wavelet coefficients such as the Gaussian [3] and Student-t [4] copulas, and the multivariate elliptical distributions.
In this paper, two elliptical distributions are considered to model the color and spatial dependencies of the wavelet coefficients: the multivariate Laplace [5] and $\mathcal{G}^{0}$ distributions. For the $\mathcal{G}^{0}$ distribution, a closed form of the geodesic distance (GD) [6] is established for fixed shape parameters and an approximation of the GD is given for the general case by assuming the geodesic coordinate functions as straight lines. A multi-model approach is then proposed for the classification of texture images. Next, some experimental results on the VisTex database are shown in section 3 to evaluate the retrieval performance. Finally, some conclusions of this work are given.

\section{MULTIVARIATE TEXTURE MODELING}

To model the spatial or color dependencies of the wavelet coefficient, various multivariate models can be considered such as copulas [3] [4]. Here, two elliptical distributions are considered: the multivariate Laplace and multivariate $\mathcal{G}^{0}$ distributions.

\subsection{The multivariate Laplace distribution}

The Multivariate Generalized Gaussian Distribution (MGGD) has been introduced in [5] to model the wavelet coefficients of color images. The Multivariate Laplace, a particular case of the MGGD, is generally considered as a reference model for color texture indexing. Its pdf is given by:

$p_{\mathbf{k}}(\mathbf{k} \mid[M])=\frac{1}{|[M]|^{\frac{1}{2}}} \frac{\Gamma\left(\frac{p}{2}\right)}{\pi^{\frac{p}{2}} \Gamma(p)} \frac{1}{2^{p+1}} \exp \left\{-\frac{1}{2}\left(\mathbf{k}^{T}[M]^{-1} \mathbf{k}\right)^{\frac{1}{2}}\right\}$

where the superscript $T$ denotes the transpose operator and $|[M]|$ is the determinant of the covariance matrix $[M] . p$ is the dimension of the vector $\mathbf{k}$. In the following, the covariance matrix $[M]$ is estimated using the method of moments [5]. 


\subsection{The multivariate $\mathcal{G}^{0}$ distribution}

To model the wavelet coefficients, other multivariate models can be considered such as the Spherically Invariant Random Vectors (SIRV). This class of models has been introduced to take into account the non-Gaussianity of the signal. In such a case, the observed vector $\mathbf{k}$ is decomposed as:

$$
\mathbf{k}=\sqrt{\tau} \mathbf{z},
$$

where $\tau$ is a scalar random variable called the multiplier $(\tau \in$ $\mathbb{R}^{+}$) and $\mathbf{z}$ a real Gaussian vector with zero mean and covariance matrix $[M]=\mathbb{E}\left\{\mathbf{z z}^{T}\right\}$. The processes $\tau$ and $\mathbf{z}$ are assumed independent.

In the literature, various models issued from the Pearson system have been introduced to represent the multiplier $\tau$. For Gamma, Inverse Gamma, Fisher, Beta and Inverse Beta distributed multiplier, the SIRV vector $\mathbf{k}$ follows respectively a $\mathcal{K}, \mathcal{G}^{0}$, KummerU, $\mathcal{W}$ and $\mathcal{M}$ distributions. An overview of those multivariate models is given in [7].

Let $\tau$ be an Inverse Gamma distributed random variable. Its pdf $p_{\tau}(\tau)$ is given by:

$p_{\tau}(\tau)=\mathcal{G} \mathcal{I}(\tau \mid m, \mathcal{M})=\frac{1}{\Gamma(\mathcal{M})} \frac{1}{\mathcal{M} m}\left(\frac{\mathcal{M} m}{\tau}\right)^{\mathcal{M}+1} e^{-\frac{\mathcal{M} m}{\tau}}$

where $\mathcal{M} \geq 0, m>0$ and $\tau \in[0,+\infty[. \mathcal{M}$ is a shape parameter while $m$ is a scale parameter. For an Inverse Gamma distributed multiplier, the vector $\mathbf{k}$ follows the $\mathcal{G}^{0}$ distribution (or Student-t), its pdf is given by [8]:

$$
\begin{aligned}
p_{\mathbf{k}}(\mathbf{k} \mid[M], m, \mathcal{M}) & =\frac{1}{(2 \pi)^{\frac{p}{2}}|[M]|^{\frac{1}{2}}} \frac{(\mathcal{M} m)^{\mathcal{M}}}{\Gamma(\mathcal{M})} \Gamma\left(\frac{p}{2}+\mathcal{M}\right) \\
& \times\left(\frac{\mathbf{k}^{T}[M]^{-1} \mathbf{k}}{2}+\mathcal{M} m\right)^{-\left(\mathcal{M}+\frac{p}{2}\right)}
\end{aligned}
$$

Let $\left(\mathbf{k}_{1}, \ldots, \mathbf{k}_{N}\right)$, be $N$ realizations of the vector $\mathbf{k}$. The Approximated Maximum Likelihood (AML) estimator of the normalized covariance matrix is the solution of the following recursive equation:

$$
[\hat{M}]_{\mathrm{FP}}=f\left([\hat{M}]_{\mathrm{FP}}\right)=\frac{p}{N} \sum_{i=1}^{N} \frac{\mathbf{z}_{i} \mathbf{z}_{i}^{T}}{\mathbf{z}_{i}^{T}[\hat{M}]_{\mathrm{FP}}^{-1} \mathbf{z}_{i}} .
$$

The existence and the uniqueness, up to a scalar factor, of the Fixed Point estimator of the normalized covariance matrix have been established, as well as the convergence of the recursive algorithm, irrespective of the initialization [9]. In this paper, the trace of the covariance matrix is normalized to $p$.

For a given covariance matrix $[M]$, the ML estimator of the multiplier for the pixel $i$ is given by:

$$
\hat{\tau}_{i}=\frac{\mathbf{k}_{i}^{T}[M]^{-1} \mathbf{k}_{i}}{p} .
$$

Once the $N$ multipliers $\tau_{i}$ are computed, the scale and shape parameters of the Inverse Gamma distribution ( $m$ and $\mathcal{M}$ ) are estimated by using the ML method [10].

\subsection{Rao geodesic distance}

The Kullback-Leibler Divergence (KLD) has been widely used in the past to compute a similarity measure between two populations. Unfortunately, up to our knowledge, for elliptically distributed random variables, no analytical expression of the KLD exists except for the degenerate Gaussian case. But, the Rao Geodesic Distance (GD) can be used instead. To this aim, two cases should be considered: when the shape parameters are fixed and when they aren't.

\subsubsection{Geodesic distance for fixed shape parameters}

For fixed shape parameters, the GD in the context of elliptical distributions is given by [6]:

$$
\begin{aligned}
& \mathrm{GD}\left(\Theta,[M]_{1} \| \Theta,[M]_{2}\right)= \\
& {\left[\left(3 b_{h}-\frac{1}{4}\right) \sum_{i=1}^{p}\left(r_{i}\right)^{2}+2\left(b_{h}-\frac{1}{4}\right) \sum_{i<j}^{p} r_{i} r_{j}\right]^{\frac{1}{2}},}
\end{aligned}
$$

where $r_{i}(i=1, \ldots, p)$ are the eigenvalues of $\ln \left([M]_{1}^{-1}[M]_{2}\right)$. Moreover, $b_{h}=-\mathbb{E}\left\{u^{2} w^{2}\right\} /(p(p+2))$ and $w=\left(\partial \ln h_{p}\right) /(\partial u)$. The pdf of $\mathrm{U}=\mathbf{k}^{T}[M]^{-1} \mathbf{k}$ is given by [6]:

$$
f_{\mathrm{U}}(u)= \begin{cases}\frac{\pi^{\frac{p}{2}}}{\Gamma(p / 2)} u^{p / 2-1} h_{p}(u) & \text { if } u>0 \\ 0 & \text { otherwise }\end{cases}
$$

where $h_{p}(\cdot)$ is the density generator:

$$
p_{\mathbf{k}}(\mathbf{k})=\frac{1}{|[M]|^{\frac{1}{2}}} h_{p}\left(\mathbf{k}^{T}[M]^{-1} \mathbf{k}\right) .
$$

For the multivariate Laplace distribution, it has been shown in [5] that $b_{h}=\frac{1}{4} \frac{p+1}{p+2}$. An analytical expression of the GD between two multivariate Laplace distributions is obtained as they do not involve shape parameters.

For the multivariate $\mathcal{G}^{0}$ distribution, one can prove that $b_{h}=\frac{1}{4} \frac{\mathcal{M}+\frac{p}{2}}{\mathcal{M}+\frac{p}{2}+1}$. Consequently, for fixed shape parameters, the GD between $\mathcal{G}^{0}$ distributions can be computed according to (7). Note that when $\mathcal{M}$ tends toward infinity, the $\mathcal{G}^{0} \mathrm{pdf}$ converges toward the Gaussian pdf and the coefficient $b_{h}=$ $1 / 4$ is retrieved [6]. Note also that it does not depend on the scale parameter $m$.

\subsubsection{Geodesic distance for variable $m$ and $\mathcal{M}$}

The GD between two multivariate $\mathcal{G}^{0}$ distributions with parameters $\left(m_{1}, \mathcal{M}_{1},[M]_{1}\right)$ and $\left(m_{2}, \mathcal{M}_{2},[M]_{2}\right)$ may be com- 
puted as follows:

$$
\begin{aligned}
& \operatorname{GD}\left(m_{1}, \mathcal{M}_{1},[M]_{1} \| m_{2}, \mathcal{M}_{2},[M]_{2}\right)=\int_{\left(m_{1}, \mathcal{M}_{1},[M]_{1}\right)}^{\left(m_{2}, \mathcal{M}_{2},[M]_{2}\right)} \mathrm{d} s \\
& =\int_{0}^{1} \sqrt{\sum_{\mu, \nu} g_{\mu \nu} \dot{\theta^{\mu}} \dot{\theta^{\nu}}} \mathrm{d} t
\end{aligned}
$$

where $\theta=\left(m, \mathcal{M}, r^{1}, \ldots, r^{p}\right)$ are the hyper-parameters of the $\mathcal{G}^{0}$ distribution and $g_{\mu \nu}$ the elements of the $(p+2) \times(p+2)$ Fisher information matrix defined by:

$$
\mathbf{G}=\left[\begin{array}{cccccc}
g_{m m} & g_{m \mathcal{M}} & g_{m i} & \cdots & \cdots & g_{m i} \\
g_{m \mathcal{M}} & g_{\mathcal{M M}} & g_{\mathcal{M} i} & \cdots & \cdots & g_{\mathcal{M} i} \\
g_{m i} & g_{\mathcal{M} i} & g_{i i} & g_{i j} & \cdots & g_{i j} \\
\vdots & \vdots & g_{i j} & \ddots & \ddots & \vdots \\
\vdots & \vdots & \vdots & \ddots & \ddots & g_{i j} \\
g_{m i} & g_{\mathcal{M} i} & g_{i j} & \cdots & g_{i j} & g_{i i}
\end{array}\right] .
$$

After some cumbersome computations, one can express the Fisher information matrix only as a function of the two shape and scale parameters:

$$
\begin{aligned}
g_{m m} & =\frac{\mathcal{M}}{m^{2}} \frac{\frac{p}{2}}{\frac{p}{2}+\mathcal{M}+1} \\
g_{m \mathcal{M}} & =-\frac{1}{m}+\frac{\left(2 \mathcal{M}+\frac{p}{2}\right)}{m\left(\frac{p}{2}+\mathcal{M}\right)}-\frac{(\mathcal{M}+1)}{m\left(\frac{p}{2}+\mathcal{M}+1\right)} \\
g_{\mathcal{M M}} & =-\frac{1}{\mathcal{M}}+\Psi(1, \mathcal{M})-\Psi\left(1, \frac{p}{2}+\mathcal{M}\right) \\
& +\frac{2}{\left(\frac{p}{2}+\mathcal{M}\right)}-\frac{(\mathcal{M}+1)}{\mathcal{M}\left(\frac{p}{2}+\mathcal{M}+1\right)} \\
g_{m i} & =-\frac{(\mathcal{M}+2)(\mathcal{M}+1) \mathcal{M}^{2}}{4 p(\mathcal{M})^{3}\left(\frac{p}{2}+\mathcal{M}+1\right)\left(\frac{p}{2}-1\right)} \\
g_{\mathcal{M} i} & =-\frac{1}{2} \frac{1}{\left(\mathcal{M}+\frac{p}{2}\right)}+\frac{(\mathcal{M}+2)(\mathcal{M}+1)}{4 p(\mathcal{M} m)^{2}\left(\frac{p}{2}+\mathcal{M}+1\right)\left(\frac{p}{2}-1\right)} \\
g_{i i} & =\frac{1}{4}\left(3 \frac{\mathcal{M}+\frac{p}{2}}{\mathcal{M}+\frac{p}{2}+1}-1\right) \\
g_{i j} & =\frac{1}{4}\left(\frac{\mathcal{M}+\frac{p}{2}}{\mathcal{M}+\frac{p}{2}+1}-1\right)=-\frac{1}{4} \frac{1}{\mathcal{M}+\frac{p}{2}+1}, i \neq j
\end{aligned}
$$

where $\Psi(1, \cdot)$ is the trigamma function. The GD is finally computed by using (10) and by assuming the geodesic coordinate functions as straight lines, i.e.

$$
\begin{cases}m(t) & =m_{1}(1-t)+m_{2} t \\ \mathcal{M}(t) & =\mathcal{M}_{1}(1-t)+\mathcal{M}_{2} t \\ r^{i}(t) & =r_{i} t\end{cases}
$$

This is only an approximation since the geodesic itself lives on the curved manifold.

\subsection{A multi-model approach}

When various models are available to represent the texture images, a multi-model approach can be considered for the classification. An image $x_{t}$ is assigned to the label $\hat{\omega}$ which maximizes the probability $p\left(x_{t} \mid \omega\right)$ :

$$
\hat{\omega}=\underset{\omega \in \Omega_{m}}{\operatorname{Argmax}} p\left(x_{t} \mid \omega\right),
$$

where $\Omega_{m}=(1, \ldots, m), m$ is the number of texture classes. If $K$ models are available, let:

$$
p\left(x_{t} \mid \omega\right)=\sum_{i=1}^{K} p\left(x_{t} \mid M_{i}, \omega\right) p\left(M_{i} \mid \omega\right),
$$

where $M_{i}$ refers to the $i^{s t}$ model. The second term of (21) is the prior probability. It characterizes the probability associated to the model $M_{i}$ for the class $\omega$. An approximation is:

$$
p\left(M_{i} \mid \omega\right) \equiv \frac{d_{M_{i}}(\omega)}{\sum_{j=1}^{K} d_{M_{j}}(\omega)},
$$

where $d_{M_{i}}(\omega)$ is the median distance between the images of the $\omega$ class and all other images for the $M_{i}$ criterion, i.e.:

$$
d_{M_{i}}(\omega)=\underset{x_{u} \in \omega, x_{v} \notin \omega}{\operatorname{median}} d_{M_{i}}\left(x_{u}, x_{v}\right) .
$$

$d_{M_{i}}\left(x_{u}, x_{v}\right)$ denotes the distance computed with the $M_{i}$ criterion between two populations $x_{u}$ and $x_{v}$.

Similarly, we define the first term of (21) as:

$$
p\left(x_{t} \mid M_{i}, \omega\right) \equiv \frac{\frac{1}{1+d_{M_{i}}\left(x_{t}, \omega\right)}}{\sum_{j \in \Omega_{m}} \frac{1}{1+d_{M_{i}}\left(x_{t}, j\right)}}
$$

where $d_{M_{i}}\left(x_{t}, j\right)=\min _{x_{u} \in j} d_{M_{i}}\left(x_{t}, x_{u}\right)$.

\section{RESULTS}

Several experiments are conducted to evaluate the performances of the proposed algorithms based on the GD between multivariate random variables. We work with the 40 texture classes from the MIT Vision Texture database. From each of these texture images of size $512 \times 512$ pixels, 16 subimages $(128 \times 128)$ are created. A dataset containing 640 texture images is finally obtained [1] [2].

In those experiments, three types of dependencies are considered (spatial, color and spatial/color). For the spatial dependency, the wavelet coefficients located around the neighborhood of the current spatial position are clustered in the random vector $\mathbf{k}^{\text {spatial }}$. For color images, the observed vector $\mathbf{k}^{\text {color }}$ contains the realizations of the wavelet coefficients for each channel of the RGB image. When dealing 


\begin{tabular}{||c|c|c|c|c|c|c||}
\hline \hline & \multirow{2}{*}{$\begin{array}{c}\text { Gaussian } \\
\text { copula }\end{array}$} & \multicolumn{5}{|c||}{ GD } \\
\cline { 5 - 8 } & Gamma & $\begin{array}{c}\text { Gaussian } \\
\text { SCM }\end{array}$ & $\begin{array}{c}\text { Gaussian } \\
\text { FP }\end{array}$ & $\begin{array}{c}\mathcal{G}^{0} \\
\text { fixed shape }\end{array}$ & $\begin{array}{c}\mathcal{G}^{0} \\
\text { linear }\end{array}$ & Laplace \\
\hline spatial & 76.82 & 71.51 & 76.99 & 77.44 & 79.38 & 73.56 \\
\hline color & 85.02 & 86.10 & 86.96 & 87.78 & 86.69 & 87.55 \\
\hline $\begin{array}{c}\text { spatial } \\
\text { \& color }\end{array}$ & 85.83 & 86.12 & 88.23 & 89.26 & 89.65 & 88.09 \\
\hline \hline
\end{tabular}

Table 1. Average retrieval rate (in \%) on the VisTex database.

with both spatial and color dependency, the random vector is $\mathbf{k}^{\text {spatial } / \text { color }}=\left[\mathbf{k}_{\mathrm{R}}^{\text {spatial }} ; \mathbf{k}_{\mathrm{G}}^{\text {spatial }} ; \mathbf{k}_{\mathrm{B}}^{\text {spatial }}\right]^{T}$. In this study, the orthogonal wavelet decomposition $(\mathrm{Nsc}=2)$ with Daubechies' filter db4 is considered, and a $3 \times 3$ neighborhood is used to take into account the spatial dependency.

Table 1 shows the average retrieval rate for the three considered types of dependencies and for various multivariate models (copula, Gaussian, $\mathcal{G}^{0}$ and Laplace). For the Gaussian copula with Gamma distributed margins [3], the symmetrized version of the Kullback-Leibler divergence has been considered. For the multivariate Gaussian model, the GD has been computed with both the sample covariance matrix (SCM) and fixed point covariance matrix (FP) estimators. Table 1 shows that indexing performances are better with the color models than with those characterizing the spatial dependency. A gain of about $10 \%$ is observed. By working on $\mathbf{k}^{\text {spatial/color }}$, a slight gain (around $1 \%$ ) is observed compared to the retrieval rates with the color models. To model the spatial dependency, the $\mathcal{G}^{0}$ distribution with the geodesic paths approximated as straight lines (GD linear) gives the best performances $(79.38 \%)$. For color texture indexing, the best results are achieved for the GD with the $\mathcal{G}^{0}$ model with fixed shape parameters and the multivariate Laplace distribution $(\approx 87.7 \%)$.

Fig. 1 shows the average classification rate as a function of the number of training samples. For four learned images, a gain of $2 \%$ is observed by merging the two multivariate models which characterize the best the spatial $\left(\mathcal{G}^{0}\right)$ and color (Laplace) dependencies (in red, 97.97\%) compared to the multivariate Laplace model which represents simultaneously the color and spatial dependencies (in pink, 95.94\%).

\section{CONCLUSION}

In this paper, a multiscale texture retrieval algorithm using the GD between elliptically distributed random variables has been proposed. The multivariate $\mathcal{G}^{0}$ and Laplace distributions have been introduced to model respectively the spatial and color dependencies of the wavelet coefficients. An analytical expression of the GD for fixed shape texture parameters and an approximation of the GD when assuming the geodesic coordinate functions as straight lines have been given for the $\mathcal{G}^{0}$ distribution. Some experiments on the VisTex database have shown that the combination of those two criteria leads to the best performances.

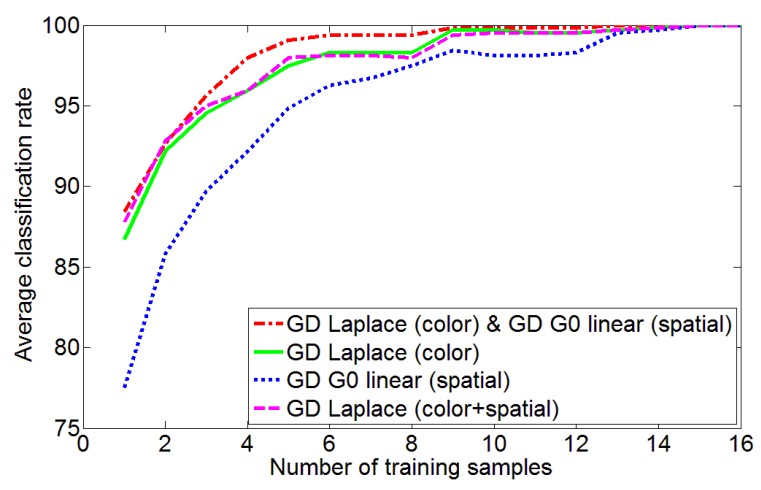

Fig. 1. Average classification rate as a function of the number of training samples.

\section{REFERENCES}

[1] M. N. Do and M. Vetterli, "Wavelet-Based Texture Retrieval Using Generalized Gaussian Density and Kullback Leibler Distance," IEEE Transactions on Image Processing, vol. 11, pp. 146-158, 2002.

[2] R. Kwitt and A. Uhl, "Lightweight Probabilistic Texture Retrieval," IEEE Transactions on Image Processing, vol. 19, no. 1, pp. 241-253, 2010.

[3] Y. Stitou, N. Lasmar, and Y. Berthoumieu, "Copulas based Multivariate Gamma Modeling for Texture Classification," in IEEE International Conference on Acoustic Speech and Signal Processing, 2009, pp. 1045-1048.

[4] R. Kwitt and A. Uhl, "A Joint Model of Complex Wavelet Coefficients for Texture Retrieval," in IEEE International Conference on Image Processing, 2009, pp. 1877-1880.

[5] G. Verdoolaege, S. De Backer, and P. Scheunders, "Multiscale Colour Texture Retrieval using the Geodesic Distance between Multivariate Generalized Gaussian Models," in IEEE International Conference on Image Processing, 2008, pp. 169-172.

[6] M. Berkane, K. Oden, and P.M. Bentler, "Geodesic Estimation in Elliptical Distributions," Journal of Multivariate Analysis, vol. 63, no. MV971690, pp. 35-46, 1997.

[7] L. Bombrun, S. N. Anfinsen, and O. Harant, "A Complete Coverage of Log-cumulant Space in Terms of Distributions for Polarimetric SAR Data," in PolInSAR, Frascati, Italy, 2011.

[8] C.C. Freitas, A.C. Frery, and A.H. Correia, "The Polarimetric G Distribution for SAR Data Analysis," Environmetrics, vol. 16, pp. 13-31, 2005.

[9] F. Pascal, Y. Chitour, J. P. Ovarlez, P. Forster, and P. Larzabal, "Covariance Structure Maximum-Likelihood Estimates in Compound Gaussian Noise : Existence and Algorithm Analysis," IEEE Transactions on Signal Processing, vol. 56, no. 1, pp. 34-48, 2008.

[10] N.L. Johnson, S. Kotz, and N. Balakrishnan, Continuous Univariate Distributions, John Wiley \& Sons, 1994. 